\title{
Adaptive Recognition of Color-Coded Objects in Indoor and Outdoor Environments
}

\author{
Yasutake Takahashi ${ }^{1}$, Walter Nowak ${ }^{2}$, and Thomas Wisspeintner ${ }^{2}$ \\ 1 Adaptive Machine Systems, Graduate School of Engineering, Osaka University, \\ Yamadaoka 2-1, Suita, Osaka, 565-0871, Japan \\ yasutake@ams.eng.osaka-u.ac.jp \\ http://www.er.ams.eng.osaka-u.ac.jp \\ 2 Fraunhofer Institute IAIS, \\ Schloss Birlinghoven, D-53754 Sankt Augustin, Germany \\ \{walter.nowak, thomas.wisspeintner\}@iais.fraunhofer.de \\ http://www.iais.fraunhofer.de
}

\begin{abstract}
To achieve robust color perception under varying light conditions in indoor and outdoor environments, we propose a three-step method consisting of adaptive camera parameter control, image segmentation and color classification. A controller for the intrinsic camera parameters is used to improve color stability in the YUV space. Segmentation is done to detect spatially coherent regions of uniform color belonging to objects in the image. Then, a probabilistic classification method is applied to label the colors by use of a Gaussian color distribution model. Experiments under combination of artificial and natural illuminations indoors and outdoors have been carried out. The results show the feasibility of this approach as well as the problems that occur under these highly diverse light situations. In particular we investigate the application in a RoboCup soccer scenario pointing toward future outdoor use.
\end{abstract}

Key words: color constancy, adaptive camera parameter control, segmentation, color classification, outdoor color vision

\section{Introduction}

Computer vision has been for long identified to provide rich information about the environment for mobile robots. One of the major challenges in interpreting camera images is to cope with influences from illumination changes. In particular color information, which humans easily can classify, may appear very differently in the camera image. This is even more the case when the robot is supposed to work in indoor and outdoor environments.

In the context of RoboCup several soccer leagues use color coded environments in well defined light conditions. One long-term goal of RoboCup is to remedy these artificial regulations and cope with natural light and objects. Yet up to now, most RoboCup teams use manually calibrated color tables and fixed 
camera parameters which have to be tuned right before the games. This tedious procedure only works when the environment does not undergo severe changes like direct sunlight and clouds during play.

We propose a combination of several techniques to approach this problem. First of all we continuously control the intrinsic camera parameters aiming for best possible color constancy. A first segmentation step based on Markov Random Fields leads to regions of uniform colors, which are then probabilistically classified to a set of discrete colors. Experiments convey that this method provides robust color classification under a variety of illumination conditions.

The paper is structured as follows. In chapter 2 we give an overview on related work regarding color constancy and color classification. Our approach is described in chapter 3 including camera parameter control, color segmentation and classification. In Chapter 4 we evaluate these steps by providing experimental results. Chapter 5 concludes with a discussion of the results and future work.

\section{Related Work}

A vast body of research has been done in the field of color constancy. Here the focus traditionally lies on the identification of illumination-independent descriptors for surfaces in a scene [1]. This includes the two tasks of determining the illuminant of a scene and mapping color values to a set of descriptors. An important instance of this general problem is the correction of colors in one image to match another image with some other illumination [2]. The available algorithms can be roughly divided into physics-based methods which try to model and explain the underlying physical processes, such as the dichromatic reflectance model, and statistics-based methods. These try to correlate distributions of colors under different illuminations, usually requiring enough colors to be present in the image. Examples are the diagonal method, gray-world methods, max-RGB [3], gamut mapping [4] as well as machine learning methods [5]. Another option is the use of chromaticity (normalized) color spaces such as YUV or HSI, where the brightness of each color is stored explicitly. It has been shown in [4] that methods using only chromaticity show similar performance as in full RGB space, but are more stable to shadows. Since the brightness also has an influence on the color value in the image due to camera characteristics and limitations, in our paper we go beyond those approaches by implementing an online method to keep the brightness in the image stable.

As several authors [6] [7] [8] point out, such algorithms have to deal with very big differences in the appereance of one color. Color regions may overlap, and the values of a set of colors change in various and highly nonlinear ways. This is particulary the case when the type of light changes, e.g. from natural to artificial light.

The application of mobile robots enables the use of online methods, such as online adaptation of camera parameters. The problem here lies in the nonlinear control and calculation of the control error. The concrete meaning of a camera 
parameter can vary much between different cameras, and is often not exactly specified. Even worse the relation between parameter value and effect is usually very non-linear. One possible remedy is to apply learning methods as in [9].

One approach for determining the required control errors is the use of reference colors. For example white can be used to set the camera's white balance parameter. Catadioptric camera systems are used by many RoboCup teams. Here a small colored ring can be laid around the camera objective so that it is always visible in the image and does not hide the view of the field itself.

Another approach is the use of semantic knowledge about the environment. This can be especially well applied in RoboCup environments due to the known field specifications. [10] and [11] first compute the pose of the robot on the field using mainly black-and-white information, then calculate the position of colored objects and finally adapt to the observed colors dynamically. [12] apply knowledge about the field and borders of objects. A comparable approach was done by [13] to recognize roads, assuming that a road is mostly flat and the car is driving on one. In our approach we avoid using such context information to account for broader application scenarios. Alternatively [14] use a three-step method to identify pixels usable as white reference; in contrast to our work they only control the white balance parameter and require white colors to be present in the environment.

Several papers investigate the benefits of first doing a segmentation or edgedectection step, and then classify the colors of whole segments. [15] and [16] use such methods with the main aims of improved color recognition and fast processing time. In [17] it was found that among different alternatives the method of choosing an unsure color (" "maybe-color"') to fit to its surrounding ones gave the best results.

Color classification by modeling color distributions as Gaussians was used by [18] and [19]. [20] shows that a discrete set of illumination conditions (bright, intermediate, dark) already improves the classification result significantly. In our paper we give further evidence for such a differentiation, as well as highlighting the benefits resulting from a continuous adaption.

\section{Process for robust color perception}

The proposed method consists of the following major processing steps:

1. segment vertical lines into regions based on spatial uniformity of color

2. calculate mean color value for each segment

3. classify each segment to a set of color representatives

4. control camera parameters using reference colors

These steps will be described in more detail in the following subsections.

\subsection{Image Segmentation}

We adopt a boundary-based Markov Random Field method for line-based segmentation of an image. Markov Random Fields have been proposed as model 

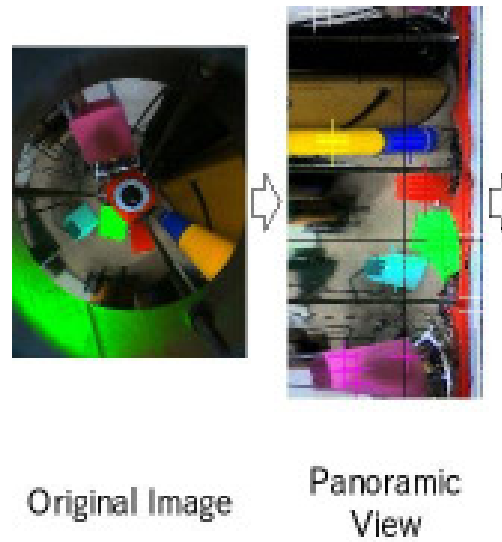

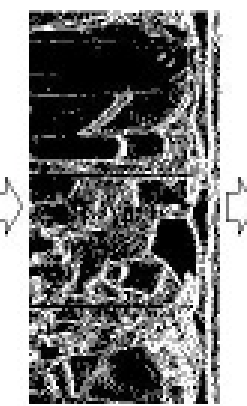

Segmentation

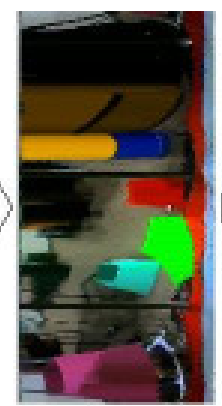

\section{Calculation of mean color} values in segments

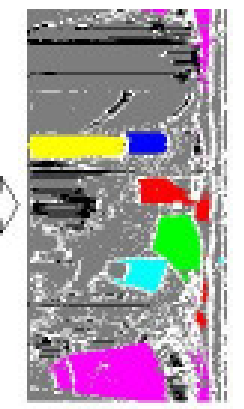

Color

Classification

Fig. 1. Image processing steps

for the visual field in the brain. Many variations of Markov Random Field have been developed, some of them have been already applied to the task of image segmentation. This method provides a sophisticated way to segment an image into spatially uniform regions. Here, we introduce the idea of the boundary-based Markov Random Field briefly.

First, we define an energy function $E(f, l \mid d)$ as follows:

$$
E(f, l \mid d)=\frac{1}{2} \sum_{i}\left(f_{i}-d_{i}\right)^{2}+\lambda \sum_{i}\left(1-l_{i}\right)\left(f_{i+1}-f_{i}\right)^{2}+\theta \sum_{i} l_{i}
$$

where $d$ is an intensity process vector representing the observed image line. Each intensity value $d_{i}$ is supposed to include some noise. $f$ is the estimated value vector. $l$ is called line process. $l_{i}$ represents the discontinuity (edge) between the $i$ th pixel and pixel $i+1$. It is 1 if it is a boundary, and 0 otherwise.

The first term of equation 1 is for data fitting and tries to minimize the error of estimation. The second term is for smoothness in space. While there is no boundary specified by the line process $l_{i}$ it tries to minimize the difference between conjunct pixels $f_{i}$ and $f_{i+1}$. When the line process $l_{i}$ is 1 , i.e. there is a boundary, then no constraint between the conjunct pixels is introduced. The third term of 1 is a constraint on the number of boundaries. This means there should be less boundaries in the image than number of pixels.

In order to minimize the energy function (1), we use a hill-climbing method and introduce derivatives of $f_{i}$ and $l_{i}$ :

$$
\begin{gathered}
\frac{\partial f_{i}}{\partial t}=\lambda\left\{\left(1-l_{i-1}\right)\left(f_{i-1}-f_{i}\right)+\left(1-l_{i}\right)\left(f_{i+1}-f_{i}\right)\right\}-\left(f_{i}-d_{i}\right) \\
\frac{\partial l_{i}}{\partial t}=-l_{i}+H\left(\frac{\lambda}{2}\left(f_{i+1}-f_{i}\right)^{2}-\theta\right)
\end{gathered}
$$


where $H(\cdot)$ is a step function. Each parameter is updated with the above derivative iteratively until it reaches convergence.

After obtaining the segmentation of the image, the mean value of each segment is calculated and used for color classification.

\subsection{Color Classification}

A probabilistic classification method based on Mahalanobis distances is applied to label colors. A Gaussian model of the color distribution for each color, consisting of mean vector and covariance matrix in YUV space must be provided beforehand. The mean color value of each segment is used to calculate the Mahalanobis distances with respect to the color distribution models. To illustrate this, one reference color is assumed to be a distribution with mean $\mu=\left(\mu_{y}, \mu_{u}, \mu_{v}\right)$ and covariance matrix $\Sigma$. The Mahalanobis distance between a color value $x=\left(x_{y}, x_{u}, x_{v}\right)$ and this distribution is defined as:

$$
D_{M}(x)=\sqrt{(x-\mu)^{T} \Sigma^{-1}(x-\mu)}
$$

Each segment is associated to the reference color with the minimal Mahalanobis distance to the segment's mean value, provided this is below a predefined threshold. This threshold value offers a way to tune the ratio between unidentified pixels and false positive ones.

\subsection{Camera Parameter Control}

To achieve color constancy under different light conditions we use a set of PID controllers to modify relevant intrinsic camera parameters. To compensate for intensity changes of the illumination, Gain and Iris are being controlled, using the mean $\mathrm{Y}$ value of a white reference color visible in the camera image.

To account for changes of the type of illumination the two White Balance channels are being controlled by using the mean $\mathrm{U}$ and $\mathrm{V}$ values of the white reference color. Furthermore, an additional red reference color is used to control Saturation in the same way using the calculated mean saturation value.

The parameters of each PID controller can be tuned by analysis of the step response switching from dark to bright illumination, from bright to dark and between different types of illumination.

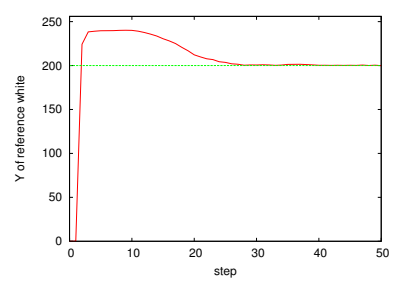

Fig. 2. Step response with optimized control parameters for the brightness controller 
Fig. 3.3 shows a step response with optimized control parameters for the Brightness controller, switching illumination from dark to bright. In this example, the controller only operates on every fifth step.

After the PID parameter optimization, a proper desired value for each controller has to be determined. This can be done by qualitative analysis of the YUV color distribution. In a distribution optimal for color classification the colors should be widely spread in the color space. On the other hand colors should not be over-saturated, i.e. the distribution should not reach the borders of the YUV cube. Furthermore the center of the distribution should lie in the center of the given YUV space.

\section{Experiments}

To evaluate the performance of our approach, we conducted several experiments in indoor and outdoor environments under different light conditions.

As basis for our experiments we used a VolksBot robot[21] with a catadioptric camera system. A variant is used in the AIS/BIT RoboCup MSL team. Processing was done on an onboard laptop with a Pentium M $1.8 \mathrm{GHz}$ processor. The full vision processing takes less then $20 \mathrm{~ms}$ for one image, depending on the number and sizes of recognized color regions. Thus the algorithm can work in real-time.

The vision system consists of a Sony DSW 500 camera looking into a hyperbolic mirror, thus producing 360 degree panoramic YUV images. A ring of white and red paper is fixed around the camera lens, see Fig.1 left. This ring provides the reference colors used by the camera parameter controller without interfering with the view of the scene itself.

The colored objects used for color classification are mainly taken from the RoboCup scenario, in particular blue and yellow goals, a green field with white lines, cyan and magenta markers, a red ball and black robots. For outdoor tests we used a subset of these.

To account for a broad range of light conditions, we regard the following situations:

1. Indoor: only artificial light of one light source (630 Lux)

2. Indoor: mixed artificial and indirect sun light (1370 Lux)

3. Indoor: only indirect sun light (500 Lux)

4. Outdoor: camera and objects in direct sun light (97,000 Lux)

5. Outdoor: camera and objects in shadow (2,550 Lux)

Fig.3 shows the camera images under these different light conditions.

\subsection{Color constancy}

Fig.4 shows the merged distributions of YUV values obtained from the color objects under the three indoor light conditions. The upper left image shows the 


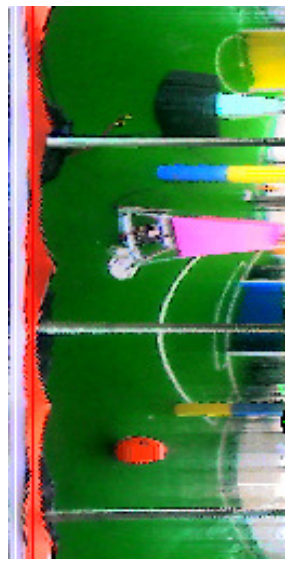

(a) indirect sun light

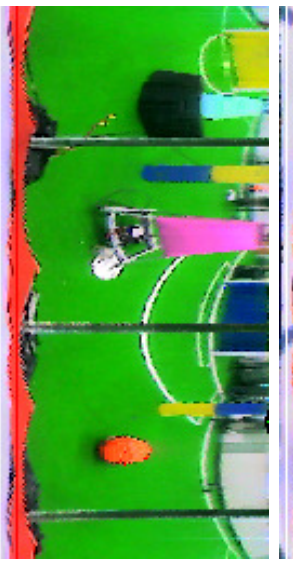

(b) artificial light

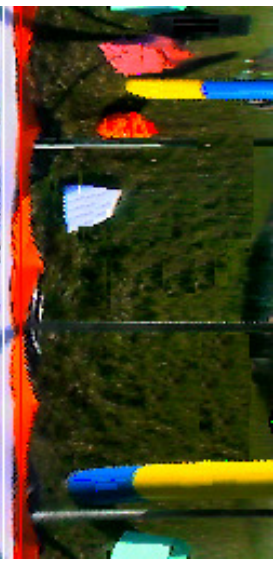

(c) sunshine

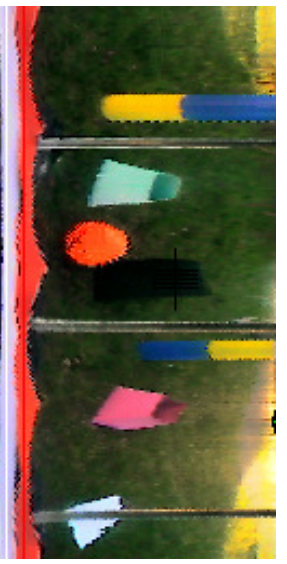

(d) shadow

Fig. 3. Captured panoramic images with PID controller under four light conditions

$3 \mathrm{D}$-view and the upper right image shows the 2D-projection on the UV-plane using PID control of the camera parameters. The lower left and right images illustrate the distributions with fixed camera parameters and embedded camera control in the UV-plane respectively.

It is shown how the color drift is greatly reduced when applying the PID controller, while the colors drift heavily for the other two approaches. Not using PID control, the drift can be so big that the color distributions overlap, making it impossible to deduce from one YUV value a unique color class.

It should be noted that also with the PID control the colors significantly drift depending on changes of direction of illumination, changes of intensity, changes of the ratio of different kinds of illumination or reflections. Still, the PID control provides better stability and spreading of the distributions compared to the other approaches evaluated. The system provides highest color constancy, when both, the object and the reference colors rings are exposed to equal light conditions.

The biggest change in color value occurs without any parameter control. It is interesting that not only the brightness $\mathrm{Y}$, but also $\mathrm{U}$ and $\mathrm{V}$ change when illumination intensity decreases. This indicates that a simple brightness normalization is not enough to identify colors robustly, giving reason to also control the saturation value of the camera.

Table 1 lists mean values and standard deviations for three object colors under diverse light conditions with different control methods for the indoor and outdoor experiments. The table only shows the standard deviation in the direction of $\mathrm{Y}, \mathrm{U}$ and $\mathrm{V}$ axes. Comparing the standard deviations of the different approaches for a certain color, like e.g. red, the lower drift of the PID control 

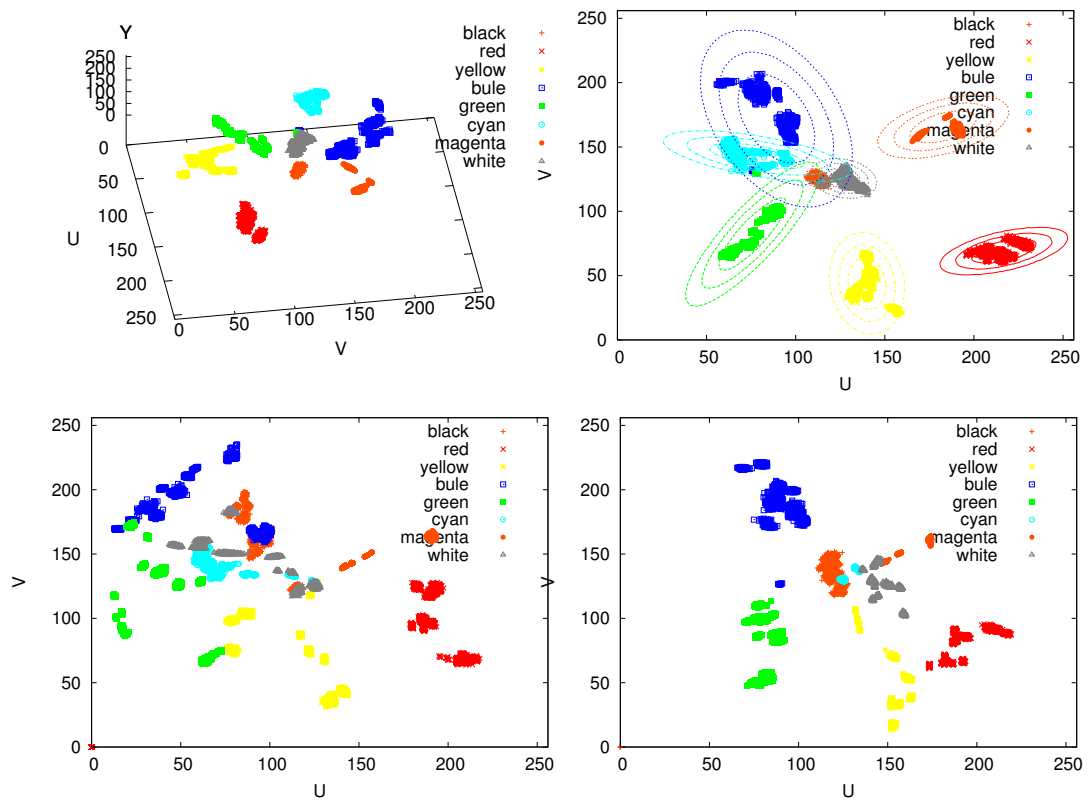

Fig. 4. Plots of YUV color distribution indoors

method can be confirmed. It is apparent that the standard deviation with PID control is nearly always smaller than for the others.

The conditions change drastically when going from indoor to natural light conditions outdoors. The image in Fig.5 shows the YUV distributions of the object colors and their projection into the UV-plane for the outdoor experiment in direct sunshine and shadow. The reason for the observable higher color drift lies in the fact of having a huge intensity range from 2550 to 97,000 Lux between shadow and direct sunlight.

Especially in the experiment undertaken in direct sunlight these extreme illumination ranges occur in a single scene, having the same objects partly exposed to direct sunshine and partly lying in its own shadow. Furthermore, the drift in color space is highly depending on the pose of the objects relative to the light source and to the camera. Also surface properties of the objects have a bigger influence here. Related to this huge illumination range, one can also see the need for the saturation control, as saturation of an object color decreases for dark and bright situations significantly.

The red color for example has a much lower saturation $\mathrm{V}$ value when the camera is outdoors. We assume that not the kind of illumination, but the high intensity and the limited color range of the camera sensor is responsible for this effect. The color is much brighter outdoors; since the YUV space is of conical shape, this results in a lower range of possible saturation values. 


\begin{tabular}{|c|rrr|rrr|rr|rr|rr|}
\hline & \multicolumn{9}{|c|}{ Indoor } & \multicolumn{3}{|c|}{ Outdoor } \\
\hline & \multicolumn{3}{|c|}{ PID } & \multicolumn{3}{|c|}{ No PID } & \multicolumn{3}{|c|}{ PID } \\
\hline red $\mu$ & 127.9 & 216.8 & 69.0 & 133.2 & 179.6 & 87.9 & 162.2 & 194.1 & 81.1 & 134.9 & 213.6 & 60.6 \\
red $\sigma$ & 15.6 & 8.9 & 4.6 & 44.6 & 53.2 & 33.2 & 17.7 & 12.6 & 10.8 & 8.8 & 6.3 & 10.1 \\
\hline yellow $\mu$ & 187.0 & 140.0 & 44.0 & 206.7 & 118.4 & 81.6 & 219.1 & 148.9 & 51.9 & 189.5 & 163.5 & 24.1 \\
yellow $\sigma$ & 26.0 & 5.2 & 9.8 & 34.8 & 21.3 & 34.1 & 20.5 & 10.1 & 30.9 & 16.3 & 7.3 & 11.5 \\
\hline blue $\mu$ & 63.1 & 89.2 & 172.1 & 98.6 & 64.3 & 191.6 & 101.4 & 89.8 & 192.0 & 83.2 & 84.1 & 186.1 \\
blue $\sigma$ & 24.1 & 5.2 & 9.8 & 43.8 & 26.8 & 23.1 & 32.2 & 8.7 & 14.0 & 22.8 & 21.6 & 23.8 \\
\hline
\end{tabular}

Table 1. means $\mu_{y, u, v}$ and standard deviations $\sigma_{y, u, v}$ of typical colors in YUV space under various light condition in indoor/outdoor environment
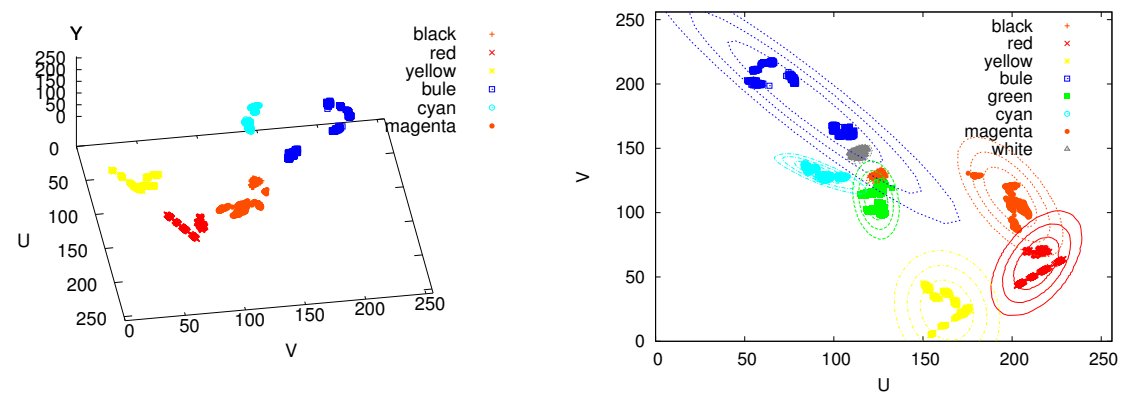

Fig. 5. YUV color distributions in outdoor environment

Still, the color distributions do not overlap, which indicates that a proper classification of colors should be possible. This will be evaluated in the next section.

\subsection{Classification Results}

First we have a look at the mean values and standard deviations of the reference color distributions, since these form the basis for the color classification step. In Fig. 4 upper right and Fig. 5 right, these regions are drawn as ellipses around the distribution of the respective colors. The images show the projection of the 3-dimensional ellipsoids on the UV-plane. The drawn ellipses represent the borders of 2- $\sigma, 3-\sigma$ and $4-\sigma$ areas. Since the ellipsoids differ in the Y-values they cover, they do actually not overlap in the way the image of their projections may suggest.

The drawing of the ellipsoids indicates what threshold to use to retrieve a binary classification result. Since the majority of already measured color pixels should be included, at least $3 \sigma$ seems reasonable. For a more robust identification towards unexpected light variations a higher value could be useful. But as this 


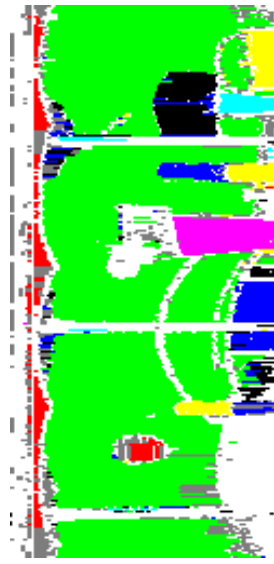

(a) indirect sun light

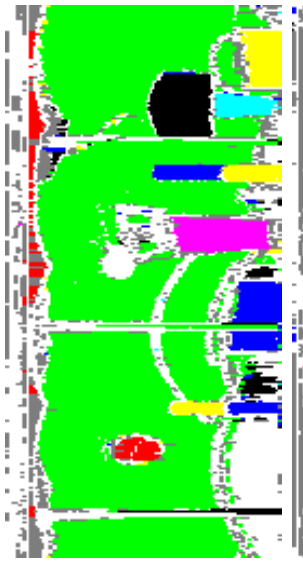

(b) artificial light

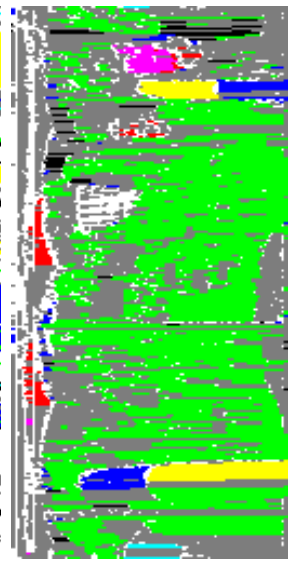

(c) sunshine

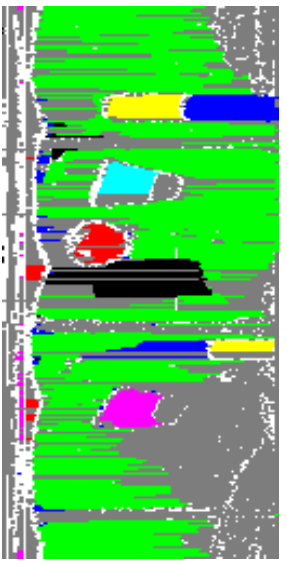

(d) shadow

Fig. 6. Classification results with PID controller in indoor and outdoor environments

can result in more false positive classifications, a compromise must be found. For our classification experiments we chose a threshold of $3 \sigma$.

Fig. 6 show the classification results in multiple light conditions. In general for all situations the classification algorithm shows a good performance. In the indoor environment all objects are recognized with their correct colors, and only very few false positive classifications exist. In the outdoor environment the method has problems with very dark pixels resulting from the high differences in intensities due to sunlight and shadow.

\section{Conclusion}

We have presented a robust color perception method including PID controller of camera parameters, segmentation by Markov Random Field, and classification based on Mahalanobis distance. The PID controller provided enough color constancy to be able to fuse the distribution under different light conditions and to generate reference color models for indoor and outdoor. These reference color models have shown to provide a robust basis for color classification under a variety of different light conditions. The big difference of color distribution in indoor and outdoor suggest the use of separate reference models for these two cases.

The vast illumination range occurring outdoors within one image has shown the physical limitations of the camera. Future work will investigate possible use of attention based mechanisms to choose from different parameter sets for different light situations. 


\section{References}

1. Maloney, L., Wandell, B.: Color constancy: A method for recovering surface spectral reflectance. J. Opt. Soc. Amer. A 3 (1986) 29-33

2. Forsyth, D.: A novel algorithm for color constancy. Int. Journal of Computer Vision 5(1) (1990) 5-36

3. Barnard, K., Funt, B., Cardei, V.: A comparison of computational colour constancy algorithms; part i: Methodology and experiments with synthesized data. IEEE Trasactions in Image Processing 11(9) (2002) 972-984

4. Finlayson, G., Hordley, S.: Improving gamut mapping color constancy. IEEE Trans. on Image Processing 9(10) (2000) 1774-1783

5. Austermeier, H., Hartmann, G., Hilker, R.: Colour-calibration of a robot vision system using self-organising feature maps. In: ICANN'96 Proc. Int Conf on Artificial Neural Networks. (1996) 257-262

6. Mayer, G., Utz, H., Kraetzschmar, G.: Playing robot soccer under natural light: A case study. In Polani, D., Browning, B., Bonarini, A., eds.: RoboCup 2003 - Robot Soccer World Cup VII. Lecture Notes in Computer Science, Berlin, Springer Verlag (July 10-11 2003) $238-249$

7. Austin, D., Barnes, N.: Red is the new black - or is it? In: Proceedings of the 2003 Australian Conference on Robotics and Automation, Brisbane, Australia. (2003)

8. Funt, B., Barnard, K., Martin, L.: Is machine colour constancy good enough? In: Computer Vision - ECCV'98. Lecture Notes in Computer Science, Springer (1998)

9. Grillo, E., Matteucci, M., Sorrenti, D.G.: Getting the most from your color camera in a color-coded world. In Nardi, D., Riedmiller, M., Sammut, C., Santos-Victor, J., eds.: RoboCup 2004: Robot Soccer World Cup VIII. Lecture Notes in Computer Science, Springer (2004) 221-235

10. Gunnarsson, K., Wiesel, F., Rojas, R.: The color and the shape: Automatic on-line color calibration for autonomous robots. In Bredenfeld, A., Jacoff, A., Noda, I., Takahashi, Y., eds.: RoboCup 2005: Robot Soccer World Cup IX. Lecture Notes in Computer Science, Springer (2005) 347-358

11. Heinemann, P., Sehnke, F., Streicher, F., Zell, A.: Towards a calibration-free robot: The act algorithm for automatic online color training. In Lakemeyer, G., Sklar, E., Sorrenti, D., Takahashi, T., eds.: RoboCup 2006: Robot Soccer World Cup X. Lecture Notes in Artificial Intelligence, Springer (2006)

12. Jüngel, M., Hoffmann, J., Lötzsch, M.: A real-time auto-adjusting vision system for robotic soccer. In Polani, D., Browning, B., Bonarini, A., Yoshida, K., eds.: RoboCup 2003: Robot Soccer World Cup VII. Lecture Notes in Computer Science, Springer (2003) 214-225

13. Thrun, S., Montemerlo, M., Dahlkamp, H., Stavens, D., Aron, A., Diebel, J., Fong, P., Gale, J., Halpenny, M., Hoffmann, G., Lau, K., Oakley, C., Palatucci, M., Pratt, V., Stang, P., Strohband, S., Dupont, C., Jendrossek, L.E., Koelen, C., Markey, C., Rummel, C., van Niekerk, J., Jensen, E., Alessandrini, P., Bradski, G., Davies, B., Ettinger, S., Kaehler, A., Nefian, A., Mahoney, P.: Stanley, the robot that won the darpa grand challenge. Journal of Field Robotics 23(9) (2006) 661-692

14. Chikane, V., Fuh, C.S.: Automatic white balance for digital still cameras. Journal for Information Science and Engineering 22 (2006) 497-509

15. de Cabrol, A., Bonnin, P., Costis, T., Hugel, V., Blazevic, P., Bouchefra, K.: A new video rate region color segmentation and classification for sony legged robocup application. In Bredenfeld, A., Jacoff, A., Noda, I., Takahashi, Y., eds.: RoboCup 2005: Robot Soccer World Cup IX (Poster Session). Lecture Notes in Computer Science, Springer (2005) 436-443 
16. Lovell, N.: Illumination independent object recognition. In Bredenfeld, A., Jacoff, A., Noda, I., Takahashi, Y., eds.: RoboCup 2005: Robot Soccer World Cup IX. Lecture Notes in Computer Science, Springer (2005) 384-395

17. Stanton, C., Williams, M.A.: A novel and practical approach towards color constancy for mobile robots using overlapping color space signatures. In Bredenfeld, A., Jacoff, A., Noda, I., Takahashi, Y., eds.: RoboCup 2005: Robot Soccer World Cup IX (Poster Session). Lecture Notes in Computer Science, Springer (2005) 444451

18. Kikuchi, T., Umeda, K., Ueda, R., Jitsukawa, Y., Osumi, H., Arai, T.: Improvement of color recognition using colored objects. In Bredenfeld, A., Jacoff, A., Noda, I., Takahashi, Y., eds.: RoboCup 2005: Robot Soccer World Cup IX. Lecture Notes in Computer Science, Springer (2005) 537-544

19. Anzani, F., Bosisio, D., Matteucci, M., Sorrenti, D.G.: On-line color calibration in non-stationary environments. In Bredenfeld, A., Jacoff, A., Noda, I., Takahashi, Y., eds.: RoboCup 2005: Robot Soccer World Cup IX. Lecture Notes in Computer Science, Springer (2005) 396-407

20. Sridharan, M., Stone, P.: Towards illumination invariance in the legged league. In Nardi, D., Riedmiller, M., Sammut, C., Santos-Victor, J., eds.: RoboCup 2004: Robot Soccer World Cup VIII. Lecture Notes in Computer Science, Springer (2004) $196-208$

21. Wisspeintner, T., Nowak, W., Bredenfeld, A.: Volksbot - a flexible componentbased mobile robot system. In Bredenfeld, A., Jacoff, A., Noda, I., Takahashi, Y., eds.: RoboCup 2005: Robot Soccer World Cup IX. Lecture Notes in Computer Science, Springer (2005) 716-723 